\title{
LA LINGUA PER NARRARE L'ASSURDO \\ Una guida alla lingua di Se questo è un nomo di Primo Levi
}

\author{
Anna Basevi \\ Doutoranda em Literatura Italiana, UFRJ \\ Bolsista CAPES \\ annabasevi@hotmail.com
}

\section{Resumo:}

É isto um homem? de Primo Levi apresenta aspectos linguísticos e literários interessantes, opções de construção do texto e referências literárias originais. A escolha dos sujeitos gramaticais confereum significado especial a uma autobiografia sobre o campo de extermínio nazista que é um testemunho coletivo antes do que pessoal. $O$ uso do tempo presente, alternado ao perfeito,como tempo privilegiado em alguns momentos reforça este objetivo, evitando qualquer efeito de ficção, mas também transmitindo e descrevendo tempo parado, dolorosamente imutável e sem esperança dos prisioneiros. $\mathrm{O}$ diálogo com a Comédia de Dante introduz elementos de forte intertextualidade fornecendo ao escritor a oportunidade de representar um mundo dominado pelo absurdo, difícil de se descrever e compreender. Com exemplosextraídos diretamente do texto, pretendemos, portanto, oferecer aos estudantes de literatura italiana um guia e um estímulo para o estudo de aspectos linguísticos e estilísticos de uma das principais obras de testemunho italianas e mundiais do século XX.

Palavras-chave: Literatura italiana. Dante. Inferno. Primo Levi. Testemunho. 


\begin{abstract}
:
Se questo è un nomo di Primo Levi presenta spunti linguistici e letterari a partire proprio dalla lingua che usa, le scelte di costruzione del testo, i riferimenti letterari. La scelta dei soggetti imprime un significato speciale a un'autobiografia che vuole essere soprattutto testimonianza di una vicenda quella dei campi di sterminio nazista - collettiva prima che personale. L'uso del presente come tempo della narrazione alternato al passatorafforza quest'obiettivo, evitando ogni effetto di finzione ma anche trasmettendo e raccontando il tempo fermo, dolorosamente immutabile e privo di speranze dei prigionieri. Il dialogo serrato con la Commedia di Dante introduce elementi di forte intertestualità che forniscono allo scrittore la possibilità di rappresentare un mondo dominato dall'assurdità e di difficile descrizione e comprensione. Con questi esempi estratti direttamente dal testo, si vuole pertanto offrire agli studenti di letteratura italiana una guida e uno stimolo allo studio di aspetti linguistici e stilistici di una delle principali opere di testimonianza italiane e mondiali del XX secolo.
\end{abstract}

Parole-chiave: Letteratura italiana. Dante. Inferno. Primo Levi. Testimonianza.

\begin{abstract}
:
If this is a Man? by Primo Levi presents interesting linguistic and literary aspects starting from the languages that he uses, the choices of construction of the text, the literary references. Sometimes, the choice of plural subjects gives a special meaning to an autobiography about nazi concentration camp that is above all a collective testimony before than personal. The use of the present and past as verbal times reinforces this objective, avoiding any effect of fiction but also transmitting and representing the immutable and hopeless
\end{abstract}


LA LINGUA PER NARRARE L'ASSURDO

Una guida alla lingua di Se questo è un uomo di Primo Levi

Anna Basevi

time of the prisoners. The dialogue with the Comedy of Dante introduces elements of strong intertextuality allowing the writer to represent a world dominated by the absurdity, that would have been difficult to describe and to understand. Through examples found directly in the text, this work wants to be for students of Italian literature a guide to the study of linguistic and stylistic aspects of one of the major works of Italian and international testimony literature of the twentieth century.

Key-words: Italian literature. Dante. Hell. Primo Levi. Testimony.

La lingua di Primo Levi (Torino, 1919-1987) suggerisce interessanti percorsi di analisi. Lo scrittore, di professione chimico, cominciò la sua avventura letteraria a partire dalla testimonianza dell'esperienza del Lager nazista, dove egli stesso era stato deportato in quanto ebreo e dove sopravvisse un anno, fino alla liberazione dell'esercito sovietico nel gennaio del ‘ 45. La terribile vicenda venne raccontata nel suo primo libro Se questo è un nomo', edito la prima volta nel ' 47 e definitivamente riconosciuto da critica e pubblico a partire dagli anni ' 60, dopo l'edizione di Einaudi del 1958 e soprattutto dopo il successo del secondo romanzo, La tregua, Premio Campiello nel 1963. Si tratta di uno dei testi al contempo di testimonianza e letterari più vivi e fecondi proprio per questa doppia natura. Dopo Auschwitz $^{2}$, la letteratura, infatti, ha visto nascere un nuovo genere: la letteratura di testimonianza.

\footnotetext{
${ }^{1}$ Si userà, a partire da ora la sigla SQU per indicare l'opera.

2 Il nome proprio del maggiore complesso di campi di concentramento e sterminio ha finito per indicare e sottintendere tutti i campi di sterminio e l'intera vicenda della persecuzione nazista.
} 
Dunque, Primo Levi testimone, come egli stesso volle sempre porsi, ma anche scrittore.

Prenderemo in considerazione aspetti linguistico-letterari dell'opera $S_{e}$ questo è un uomo che paiono centrare particolarmente bene l'obiettivo di comunicare con il lettore, di raccontare in maniera efficace l'esperienza del campo di sterminio nazista .

Per quanto riguarda la struttura, il libro è diviso in 17 capitoli, divisi in parte secondo la linea cronologica degli eventi, in parte con un criterio tematico, concentrando di volta in volta aspetti specifici come il viaggio, l'infermeria, il lavoro, le qualità umane per sopravvivere, alcuni episodi o personaggi degni di analisi (Il viaggio, Ka-Be, Le nostre notti, Il lavoro, Sommersi e Salvati, Il canto di Ulisse, L'ultimo ecc.). Solo l'ultimo capitolo, "Storia di dieci giorni ", segue un filo diaristico, come se fosse stato scritto giorno dopo giorno.

Si deve a Pier Vincenzo Mengaldo il primo studio approfondito sulla lingua e la scrittura di Levi e ad Alberto Cavaglion il lavoro di commento al testo in importanti e arricchenti note all'ultima edizione del testo (Einaudi, 2012), sottolineando anche gli aspetti intertestuali.

Come hanno messo in lucei numerosi critici italiani - lungo anni in cui la fortuna critica delloscrittore non ha fatto che aumentare, specie dopo la sua morte - le caratteristiche principali della scrittura di Levisono la chiarezza, la precisione, la sobrietà, la tendenza all'economia e all'essenzialità linguistica, ma anche l'intreccio dei generi testuali, l'ironia, le metafore sia letterarie che scientifiche.Se da un lato l'intento del testimoniare si rispecchia nella ricerca della massima trasparenza, troviamo anche un forte gusto per l'oralità, un vivo 
LA LINGUA PER NARRARE L'ASSURDO

Una guida alla lingua di Se questo è un uomo di Primo Levi

Anna Basevi

senso della socialità ${ }^{3}$ della lingua che non significa lessico della lingua parlata e dei registri bassi, bensiil piacere del raccontare, quindi la volontà di rendere, appunto, chiari gli enunciati, uso di appelli diretti al lettore o di costruzioni sintattiche prive di verbo e così via.Senza dubbio, domina su tutto il testo, però, un tono alto, di lingua a tratti aulica e in genere colta.

A proposito del registro colto, Mengaldo afferma che:

La lingua aulica costituisce il "basso continuo" della prosa di Levi, in particolare là dove deve raccontare vicende o personaggi picareschi, ai quali dona un carattere inatteso di solennità, la medesima solennità che usa quando, in SQU, deve parlare di aspetti gravi e difficili. (MENGALDO, p. 170).

Marco Belpoliti, altro importante studioso dello scrittore e curatore delle OpereComplete, aggiunge, parlando di tutta la sua produzione:

C'è nella prosa di Levi un doppio movimento: da un lato
un'impostazione aulica, fondata sul modulo avverbiale di chiara
derivazione scolastica (la lingua dei licei classici, ma anche quella della
"prosa d' arte"); e dall'altro una lingua innovativa di provenienza tecnica
e scientifica su cui Levi ha particolarmente insistito e di cui è fortemente
consapevole. (BELPOLITI, Marco. Primo Levi. Milano: Mondadori,
1998, p. 107).

\section{Il lessico per descrivere la Babele}

"La confusione delle lingue è una componente fondamentale del modo di vivere di quaggiù;si è circondati da una perpetua Babele, in cui tutti urlano ordini e minacce in lingue mai prima udite, e guai a chi non afferra a volo"”.

\footnotetext{
${ }^{3}$ MENGALDO, Pier Vincenzo. Lingua e scrittura in Levi (vedere riferimenti bibliografici).

${ }^{4}$ SQU, p. 29
} 
Queste parole descrivono la percezione di Primo Levi nel trovarsi catapultato ad Auschwitz-Monowitz e destinato al lavoro forzato che si svolge nell'area denominata Buna.

La Buna è grande come una città; vi lavorano, oltre ai dirigenti e ai tecnici tedeschi, quarantamila stranieri, e vi si parlano quindici o venti linguaggi. Tutti gli stranieri abitano in vari Lager, che alla Buna fanno corona (...) e noi siamo gli schiavi degli schiavi a cui tutti possono comandare (SQU, p. 61)

In questa Babele non esiste solo la sovrapposizione di nazionalità e lingue ma anche un nuovo gergo del campo, che assimila e rimpasta diverse espressioni.

Del tedesco Levi ci offre diversi esempi lessicali, tra scritte, ordini e vocaboli vari per indicare luoghi, cose, azioni, funzioni, numeri (fondamentali per riconoscervi il proprio numero tatuato sostitutivo del nome) o frasi complete che rimangono scolpite come un avviso (Hier es gibt kein warum - Qui non c' è nessun perché ${ }^{5}$. L'autore inserisce varie forme di questo lessico in quasi ogni pagina a volte senza bisogno di traduzione immediata, altre subito spiegate. Il polacco e l'yiddish sono le seconde lingue del campo, ma verso gli ultimi mesi l'ungherese si aggiungerà alle lingue più parlate per via dell'arrivo dei prigionieri dall'Ungheria.

Il gergo che si va creando è un impasto di varie espressioni, che include anche altre lingue minoritarie se il gruppo parlante possiede una compattezza o delle caratteristiche speciali, come è il caso dei greci.

Se il polacco "Wstavac" rappresenta la sveglia mattutina, lo spagnolo "caravana" può ritrovarsi a essere la gamella e l'idea generica di furto si esprime nel greco "klepsi-klepsi", grazie alla piccola ma determinante colonia di greci bilingue di Salonicco.

\footnotetext{
${ }^{5}$ Ibid., p. 21
} 
LA LINGUA PER NARRARE L'ASSURDO

Una guida alla lingua di Se questo è un nomo di Primo Levi

Anna Basevi

La parole per pane, Brot- Broit-chleb-pain-lechem-kenyér testimoniano dell' universo mistilingue del Lager ${ }^{6}$.

La Torre del Carburo, che sorge in mezzo alla Buna e la cui sommità è raramente visibile in mezzo alla nebbia, siamo noi che l'abbiamo costruita. I suoi mattoni sono stati chiamati Ziegel, briques, tegula, cegli, kamenny, bricks, téglak, e l'odio li ha cementati; l'odio e la discordia, come la Torre di Babele, e così noi la chiamiamo: Babelturm, Bobelturm. (SQU, p. 61).

Nella Babele, dunque, fin da subito è necessario imparare più o meno rapidamente "a rispondere 'Jawohl', a non fare mai domande, a fingere sempre di aver capito"?

Il verbo imparare è uno dei principali per la sopravvivenza:imparare le lingue e il tedesco in primo luogo. Levi racconta di aver supplicato un compagno alsaziano di dargli veloci lezioni di tedesco in cambio di pane nei pochissimi intervalli a disposizione tra le fatiche e la routine micidiale del campo. Imparare il tedesco e principalmente quel "Lagerjargon",ovvero un tedesco urlato, di ordini, minacce e rudezza e la cui comprensione garantisce un primo stadio di sopravvivenza. Stadio che la maggior parte degli Italiani non ha nemmeno raggiunto: sono morti quasi tutti nelle prime due settimane per non aver capito ordini e informazioni.

I ben noti ebrei italiani, arrivati due mesi fa, tutti avvocati, tutti dottori, erano più di cento e già non sono che quaranta, quelli che non sanno lavorare e si lasciano rubare il pane e prendono schiaffi dalla mattina alla sera; i tedeschi li chiamano "zwei linke Hände" (due mani sinistre), e perfino gli ebrei polacchi li disprezzano perché non sanno parlare yiddish. (SQU, p. 39).

\footnotetext{
${ }^{6}$ SQU, p. 30

${ }^{7}$ Ibid., p. 24
} 
LA LINGUA PER NARRARE L'ASSURDO

Una guida alla lingua di Se questo è un nomo di Primo Levi

Anna Basevi

\section{La voce di chi narra}

Benché la voce narrante corrisponda alla prospettiva di chi vede ${ }^{8}$ come in ogni autobiografia, spesso predomina la scelta di introdurre il soggetto in prima persona plurale: un noi che agli inizi si riferisce a un generico e implicito "quelli che sanno", ma anche, poche pagine dopo a " un comune campione di umanità" 10 o più spesso a un noi collettivo corrispondente ai più vicini compagni di prigionia o ai prigionieri ebrei del Lager in generale. Interessante è questo uso sia nel raccontare fatti concreti che hanno riguardato tutti:

Siamo scesi, ci hanno fatto entrare in una camera vasta e nuda ${ }^{11}$

sia stati fisiologici e condizioni che si suppone tutti provassero :

Che sete abbiamo! 12

sia stati d'animo che l'autore estende agli altri, ampliando tale scelta stilistica:

Per noi la storia si era fermata ${ }^{13}$

O ancora:

poiché siamo tutti, per qualche ora, sazi, (...) ci sentiamo buoni (...) e siamo capaci di pensare alle nostre madri e alle nostre mogli, il ché di solito non accade. Per qualche ora, possiamo essere infelici alla maniera degli uomini liberi. (SQU, p. 65).

Un uso, dunque, del noi che affianca l'io permeando tutto il testo di un valore aggiunto di testimonianza poiché il testimone, in questo caso, è non solo il "superstes" 14 , cioè chi ha attraversato in prima persona un' esperienza

\footnotetext{
8 "Voce e prospettiva"secondo GENETTE, Gerard. Figure III. Torino: Einaudi, 1976

9 "Soltanto una minoranza di ingenui e illusi si ostinano nella speranza: noi avevamo parlato a lungo coi profughi polacchi e croati, e sapevamo che cosa voleva dire partire". (SQU, p. 8)

10 “e noi non eravamo che un comune campione di umanità". (SQU, p. 11)

${ }^{11}$ SQU, p. 15

${ }^{12}$ Ibidem

${ }^{13}$ Ibid., p. 102

${ }^{14}$ Concetti di testes e superstes analizzati in AGAMBEM, Giorgio. Quel che resta di Auschwitz.Milano: Bollati Boringhieri, 1998
} 
LA LINGUA PER NARRARE L'ASSURDO

Una guida alla lingua di Se questo è un nomo di Primo Levi

Anna Basevi

e la racconta, ma colui che parla degli altri, come un "testes", ovvero chi ha visto un fatto accadere a terzi ${ }^{15}$. E potremmo aggiungere che il dilemma di Levi o il suo paradosso, come lo definì Agambem ${ }^{16}$, è stato quello di volere e sentire di non potere completamente testimoniare per i sommersi, per una parte del noi raccontato corrispondente a chi non è tornato.

È interessante notare, però, che quando si tratta di parlare dei cosiddetti "muselmänner" - sorta di paria del Lager da tutti allontanati perché privi di ogni parvenza vitale e rassegnati al crollo - Levi, consapevole della distinzione fra questo tipo di sommersi e altri che hanno resistito fino all'ultimo, smette di usare il noi e usa un distaccato, ma non per questo privo di umana pietà, "essi”.

Nel gergo del campo vengono così chiamati coloro che

Hanno seguito il pendio fino in fondo (...)sono stati sopraffatti prima di aver potuto adeguarsi (...) la loro vita e` breve , ma il loro numero è sterminato (...)sono loro, i Muselmanner, i sommersi, il nerbo del campo.(...) i non-uomini (...) già troppo vuoti per soffrire veramente. Si esita a chiamarli vivi: si esita a chiamar morte la loro morte, davanti a cui essi non temono perché sono troppo stanchi per comprenderla. (SQU, p. 77-78).

E sono coloro in beneficio dei quali farsi testimoni è impresa più ardua, apparentemente vana, eppure urgente.

Se ogni opera letteraria è rivolta fuori di sé ${ }^{17}$, cioè verso l'ascoltatorelettore, SQU risponde puntualmente a questa caratteristica e si rivolge esplicitamente a un voi, lo stesso dell'epigrafe iniziale:

Voi che vivete sicuri

\footnotetext{
${ }^{15}$ Cfr. LEVI, Primo. I sommersi e i salvati.Torino: Einaudi, 2007 (1 ed.1986) p. 65

${ }^{16}$ Ibidem

${ }^{17}$ Cfr. BACHTIN Michail. Estetica e Romanzo. Torino: Einaudi, 1998
} 
LA LINGUA PER NARRARE L'ASSURDO

Una guida alla lingua di Se questo è un uomo di Primo Levi

Anna Basevi

Nelle vostre tiepide case,

Voi che trovate tornando a sera

Il cibo caldo e visi amici:

Considerate se questo è un uomo

Il rivolgersi ai lettori si ripete nelle pagine del libro, sempre come invito alla riflessione:"Non fareste voi altrettanto?Se dovessero uccidervi domani col vostro bambino, voi non gli dareste oggi da mangiare?"18

Una sola volta (ma di particolare interesse) troviamo un voi specificamente riferito ai tedeschi, in cui la parola scritta ripercorre le parole del pensiero sorto nel momento narrato. Si tratta di un episodio in cui $\mathrm{i}$ prigionieri erano stati costretti ad assistere allimpiccagione di un uomo che aveva tentato di ribellarsi, e l'avevano fatto in un silenzio rassegnato che l'autore ricorda con amarezza e angoscia:"Distruggere l'uomo è difficile quasi quanto crearlo: non è stato agevole, non è stato breve, ma ci siete riusciti, tedeschi. Eccoci docili sotto i vostri sguardi: da parte nostra nulla piè avete a temere" ${ }^{\prime 19}$. Quest'episodio segna uno dei momenti di maggiore sconforto e perdita di speranza della narrazione, il fondo della vergogna umana, in cui il titolo-domanda Se questo e` un nomo? sembra non trovare risposta, o meglio rispondere negativamente, sia che si pensi ai nazisti sia ai prigionieri.

Vorrei poter raccontare che di fra noi, gregge abietto, una voce si fosse levata, un mormorio, un segno di assenso. Ma nulla è avvenuto.Siamo rimasti in piedi, curvi e grigi, a capo chino (...)

I russi possono ormai venire: non vi sono piu uomini fra noi (...) non troveranno che noi domati, noi spenti,degni ormai della morte inerme che ci attende. (...) Alberto ed io siamo rientrati in baracca, e non abbiamo potuto guardarci in viso. (...)Abbiamo issato la menaschka sulla

\footnotetext{
${ }^{18}$ SQU, p. 9

${ }^{19}$ Ibid., p. 130
} 
LA LINGUA PER NARRARE L'ASSURDO

Una guida alla lingua di Se questo è un uomo di Primo Levi

Anna Basevi

cuccetta, abbiamo fatto la ripartizione, abbiamo soddisfatto la rabbia quotidiana della fame, e ora ci opprime la vergogna. ${ }^{20}$

È interessante notare che queste disperate parole chiudono il penultimo capitolo intitolato "L'ultimo" che è comunque l'ultimo capitolo strutturato a tema, mentre segue, poi, il capitolo già citato, a forma di diario "Storia dei dieci giorni" (che racconta la sopravvivenza nella Auschwitz abbandonata dai nazisti) ${ }^{21}$. Con la parola (e il sentimento) "vergogna" si chiude dunque il racconto della grande macchina dello sterminio creata dai tedeschi. Inoltre si ribadisce come questo abnorme "esperimento" di distruzione sia in parte riuscito. È davvero il fondo toccato dall'umanità, un'umanità che non esiste più. Non esistono più altri soggetti al di fuori del "noi, gregge abietto" e del voi, tedeschi. Infatti, dopo la condanna all'impiccagione di un uomo eseguita nel silenzio e tra gli sguardi umiliati dei prigionieri, Levi e il suo amico Alberto tornano nella baracca-dormitorio oppressi dalla mancata possibilità di ribellarsi, cosa che "l'ultimo" aveva fatto, dimostrando anche di fronte alla forca di essere l'ultimo esemplare di essere umano non sottomesso. Come si nota, all'interno della stessa frase si passa dal passato prossimo al presente per dire il senso della vergogna, rinnovandone l'attualità e perciò universalizzandola e ritrasmettendola a ogni lettore in ogni epoca.

L'avverbio "ora" potrebbe denotareil tempo ogni volta riscattato dalla memoria, un "adesso" che ci riguarda tutti.

\footnotetext{
${ }^{20}$ Ibidem

${ }^{21}$ Primo Levi si trovava in infermeria nel momento in cui i tedeschi decisero di abbandonare il Lager portandosi dietro, nelle famose marce della morte, i prigionieri in grado di camminare. Egli, perciò, rimase nel campo fino all' arrivo dei sovietici, mentre il suo amico fraterno Alberto perì durante la marcia in circostanze ignote.
} 


\section{Il tempo del testimone: uso dei tempi verbali}

È risaputo che nelle autobiografie un tempo alternativo al passato remoto - tipico tempo del romanzo tradizionale - può spesso essere il presente storico. Infatti, è il presente che conferisce un effetto di omodiegetizzazione $^{22}$, fatto questo che potrebbe non essere necessario, trattandosi di un' autobiografia, ma che viene a rafforzare la presenza dell'autore in quanto protagonista e, in questo caso, accresce l'effetto di testimonianza. Nel romanzo tradizionale, in genere, è il passato remoto a mettere in luce "una specie di passato senza età"23 - almeno per quanto riguarda lingue come l'italiano o il francese - poiche "il preterito epico non ha nessun valore temporale: segnala la finzionalità della finzione". ${ }^{24}$ Dunque, scegliendo di evitarlo, lo scrittore si è liberato da ogni residuo di effetto di finzione per puntare dritto sull'obiettivo del testimoniare.

Ma il presente usato da Levi non si limita a corroborare la voce del testimone. Esso riflette le caratteristiche del tempo percepito nel Lager: un tempo statico, stagnante, fuori dal tempo del mondo, appartenente a una dimensione altra, come un tempo di un universo sospeso tra l'oblio e il presente:

(...) così concrete la fame e la desolazione, e così irreale tutto il resto, che non

pareva possibile che veramente esistesse un mondo e un tempo, se non il nostro

mondo di fango, e il nostro tempo sterile e stagnante a cui eravamo oramai

\footnotetext{
${ }^{22}$ Cfr. GENETTE, Gerard. Nuovo discorso del racconto.Torino:Einaudi, 1990

${ }^{23}$ Ibidem

${ }^{24}$ Ibidem
} 
LA LINGUA PER NARRARE L'ASSURDO

Una guida alla lingua di Se questo è un nomo di Primo Levi

Anna Basevi

incapaci di immaginare una fine ${ }^{25}$. Per gli uomini vivi le unità del tempo hanno

sempre un valore, il quale è tanto maggiore, quanto più elevate sono le risorse internedi chi le percorre; ma per noi, ore, giorni e mesi si riversavano torpidi dal futuro nelpassato, sempre troppo lenti, materia vile e superflua di cui cercavamo di disfarci alpiù presto. (SQU, p. 101102).

Un tempo svuotato di passato quanto di futuro:

Tale sarà la nostra vita. Ogni giorno, secondo il ritmo prestabilito, Ausrückcn ed

Einrücken, uscire e rientrare; lavorare, dormire e mangiare; ammalarsi, guarire o

morire. E fino a quando? Ma gli anziani ridono a questa domanda: a questa domanda

si riconoscono i nuovi arrivati. Ridono e non rispondono: per loro, da mesi, da anni,

il problema del futuro remoto è impallidito, ha perso ogni acutezza, di fronte ai benpiù urgenti e concreti problemi del futuro prossimo: quanto si mangerà oggi, se

nevicherà, se ci sarà da scaricare carbone. (SQU, p. 27).

A proposito del tempo bloccato, Belpoliti ne sottolinea l'idea di staticità più che di eternità affermando che "il tempo della testimonianza è per Levi un tempo immobile, riferito a un qui e ora, non inteso come eterno presente (il tempo circolare del mito), bensì un tempo bloccato". ${ }^{26}$

Potremmo anche spingere ulteriormente le ipotesi fino a chiederci se il presente non possa servire da monito nei confronti di un orrore che potrebbe riproporsi nella storia umana. E l'uso del presente riporterebbe nel

\footnotetext{
${ }^{25}$ Il neretto è nostro, qui come più avanti.

${ }^{26}$ BELPOLITI, op. cit.,p. 110
} 
LA LINGUA PER NARRARE L'ASSURDO

Una guida alla lingua di Se questo è un nomo di Primo Levi

Anna Basevi

nostro tempo di lettori ciò che non dobbiamo dimenticare cosi come abbiamo accennato a proposito dell'attualita del sentimento di vergogna.

Lo conferma Mengaldo:

L'effetto di attualizzazione e drammatizzazione dei fatti narrati, quasi un portare l'autore, e il lettore, sul luogo, è connesso al valore tipicamente “astanziale"del presente storico; ma questa attualizzazione e astanzialità vengono ad assumere nel nostro caso un significato più individuato, e inquietante, come se ci venisse suggerito che Auschwitz non è esperienza che l'autore e nessuno possa ritenere esaurita, circoscritta in un tempo trascorso: il presente è il presente del suo incombere come una realtà incancellabile, che ci avvolge ancora e sempre può rinascere. (MENGALDO, p. 203).

\section{Inferno dantesco, metafora del lager}

Uno degli aspetti principali di SQU è senz'altro il dialogo che instaura con l'Inferno dantesco. L'opera infatti è permeata dall'influenza di Dante sia sul piano lessicale che sul piano dell'immaginario.

L'inferno dantesco si pone come riferimento esplicito nel descrivere situazioni infernali, personaggi diabolici e satanici, cerberi e caronti, ma anche come spazio di discesa pericolosa in cui è facile perdersi.

Come è stato detto, per LeviDante non è, comunque, un modello, ma più semplicemente una materia. ${ }^{27}$

Egli stesso nel rispondere alla domanda sul perché non avesse messo la Divina Commedia (come anche Leopardi e Manzoni) tra le opere della sua personale antologialetteraria intitolata $L$ a ricerca delle radici, risponde: "Se li

\footnotetext{
${ }^{27}$ RASTIER François, Ulisse ad Auschwitz. Primo Levi, il superstite.Napoli: Liguori, 2009, p. 52.
} 
avessi messi, sarebbe stato come se, in un documento di identità, sul rigo 'segni particolari' si scrivesse: 'due occhi' ",28.

Troviamo prestiti, citazioni, personaggi, uso di invenzioni e concetti danteschi come metafore, e descrizioni intensificate dalla somiglianza di certipaesaggi caratterizzati da buio, fango, freddo, realmente vissuti dai prigionieri del Lager polacco.

Potremmo dire, infine, che Primo Levi rappresenta per noi allo stesso tempo Dante che visita gli inferi e ci racconta questo mondo rovesciato (il Levi narratore-testimone, dallo sguardo osservatore, "antropologico" e che poi scrive), i dannati (il Levi prigioniero-schiavo) e Virgilio (il narratore che ci guida, ci spiega e ci illustra i vari personaggi e i vari gironi ), benché su queste figure e le loro relative corrispondenze ogni studioso possa rintracciare differenti combinazioni.

Ricordando che molti critici hanno sottolineato l'intertestualità con Dante, da Cesare Segre in uno dei primi saggi sul tema ${ }^{29}$ al già citato Alberto Cavaglion (per nominarne appena due tra i tanti), può essere utile presentare una specie di inventario dei legami esistenti tra il testo dantesco e SQU.

\section{a) Quali parole per descrivere}

Iniziamo con la premessa concettuale che Levi pone al suo tentativo di testimoniare: sarà possibile farlo, e con quali parole?

Allora per la prima volta ci siamo accorti che la nostra lingua manca di parole per esprimere questa offesa, la demolizione di un uomo(SQU, p. 19).

\footnotetext{
${ }^{28}$ LEVI apud BELPOLITI, op. cit. p. 65

${ }^{29}$ SEGRE, Cesare. Lettura di "Se questo è un uomo". In FERRERO, op. cit., p. 66
} 
LA LINGUA PER NARRARE L'ASSURDO

Una guida alla lingua di Se questo è un nomo di Primo Levi

Anna Basevi

Noi diciamo "fame", diciamo "stanchezza", "paura" e "dolore", diciamo "inverno", e sono altre cose. Sono parole libere create e usate da uomini liberi (...)Se i Lager fossero durati più a lungo, un nuovo aspro linguaggio sarebbe nato; e di questo si sente il bisogno per spiegare cosa è faticare l'intera giornata nel vento, sotto zero, con solo indosso camicia, mutande, giacca e brache di tela, e in corpo debolezza e fame e consapevolezza della fine che viene.(p. 107).

È il carattere estremo dell'esperienza a inoculare il dubbio sull'efficacia della testimonianza. Anche nel caso, pur immaginario, di Dante:

S'io avessi le rime aspre e chiocce,/ come si converrebbe al tristo buco/ sovra 'l qual pontan tutte l'altre rocce,/ io premerei di mio concetto il suco/ più pienamente; ma perch'io non l'abbo,/ non sanza tema a dicer mi conduco;/ ché non è impresa da pigliare a gabbo/ discriver fondo a tutto l'universo (If XXXII, vv.1-8).

b) Questo e' l'Inferno

Per quanto riguarda l'immaginario, impera in tutta l'opera l'idea inevitabile dell'inferno ed è questo che induce l'italiano Levi a leggere Auschwitz con gli occhi di Dante .

Oltre a numerose espressioni comediavoli, diabolici, demonio, satanica conoscenza, che troviamo nel corso della lettura, la natura infernale del luogo e della situazione è esplicitata fin dall'arrivo:

Ora dopo ora, questa prima lunghissima giornata di antinferno volge al termine. Mentre il sole tramonta in un vortice di nubi sanguigne (p. 21) ${ }^{30}$

Questo è l'inferno. Oggi, ai nostri giorni, l'inferno deve essere così, una camera grande evuota, e noi stanchi di stare in piedi, e c'è un rubinetto che gocciola e l'acqua non sipuò bere, e noi aspettiamo qualcosa di certamente terribile e non succede niente econtinua a non succedere niente. Come pensare? Non si può più pensare, è

\footnotetext{
${ }^{30}$ Le citazioni di questo paragrafo sono indicate solo con la pagina quando provengono da SQU. Il neretto è nostro.
} 
LA LINGUA PER NARRARE L'ASSURDO

Una guida alla lingua di Se questo è un uomo di Primo Levi

Anna Basevi

comeessere già morti. Qualcuno si siede per terra. Il tempo passa goccia a goccia. (p. 15).

ci sentivamo ormai dall'altra parte. (p. 14).

questo complicato mondo infero. (p. 16).

noi, non più vivi, noi già per metà dementi nella squallida attesa del niente. (p. 30).

Inoltre la forma a imbuto che inghiotte Dante nelle viscere della terra in un viaggio verticale verso il basso rimane la metafora visiva più forte e immediata per la sensazione di essere caduti in fondo all'abisso dell'umano. La stessa parola "fondo" è usata da Levi almeno sei volte, nonché si presta a titolo del secondo capitolo subito dopo "Il viaggio". Dunque il viaggio porta direttamente al fondo.

...vagoni merci,chiusi dall'esterno e dentro uomini donne bambini, compressi senza pietà, (...) in viaggio verso il nulla, in viaggio all'ingiù, verso il fondo.(p. 10)

Eccomi dunque sul fondo. A dare un colpo di spugna al passato

si impara assai presto, se il bisogno preme. (p. 28)

Il fondo di Levi, perciò, va letto in una prospettiva intertestuale come sarebbe importante ricordare ai traduttori di Se questo è un nomo in altre lingue. L'arrivo nel primo cerchio segna l'incontro con una figura che minacciosamente emette il giudizio e la sentenza di condanna. In Dante si tratta di Minosse che determina il numero di cerchi da scendere:

Così discesi del cerchio primaio/ giù nel secondo, che men loco cinghia/ e tanto più dolor che punge a guaio./ Stavvi Minòs orribilmente e ringhia: esamina le colpe ne l'intrata; giudica e manda secondo ch'avvinghia./ Dico che quando l'anima mal nata/ li vien dinanzi tutta si confessa:/e quel conoscitor de le peccata/ vede qual loco d'inferno è da essa; cignesi con la coda tante volte/ quantunque gradi vuol che giù sia messa./ Sempre dinanzi a lui ne stanno molte:/ vanno a vicenda ciascuna al giudizio, dicono e odono e poi son giù volte. (If $\mathrm{V}, \mathrm{vv} .1-15$ ). 
LA LINGUA PER NARRARE L'ASSURDO

Una guida alla lingua di Se questo è un nomo di Primo Levi

Anna Basevi

Nel caso di Levi, i prigionieri non hanno peccati da confessare ma, quando interpellati, rivelano professioni, età e malattie, mentre sinistra e destra determinano la selezione tra vita nel Lager ola camera a gas immediata. La scena dell'arrivo del treno blindato si apre con suoni altrettanto spaventosi che ricordano il Minosse che ringhia:

Venne a un tratto lo scioglimento. La portiera fu aperta con fragore, il buio echeggiò di ordini stranieri, e di quei barbarici latrati dei tedeschi quando comandano, che sembrano dar vento a una rabbia vecchia di secoli. Ci apparve una vasta banchina illuminata da riflettori.Poco oltre, una fila di autocarri. Poi tutto tacque di nuovo. Qualcuno tradusse:bisognava scendere coi bagagli, e depositare questi lungo il treno. In un momento labanchina fu brulicante di ombre(...). Una decina di SS stavano in disparte, l'aria indifferente,piantati a gambe larghe. A un certo momento, penetrarono fra di noi, e, con vocesommessa, con visi di pietra, presero a interrogarci rapidamente, uno per uno, incattivo italiano. Non interrogavano tutti, solo qualcuno. 'Quanti anni? Sano omalato?' e in base alla risposta ci indicavano due diverse direzioni $[\ldots]$ In meno didieci minuti tutti noi uomini validi fummo radunati in un gruppo. Quello che accaddedegli altri, delle donne, dei bambini, noi non potemmo stabilire allora né dopo: lanotte li inghiottì, puramente e semplicemente. (p. 12-13).

La notte che inghiotte i deportati fa eco, inoltre, ai versi che descrivono un primo paesaggio similmente nebuloso, metafora della perdita di orientamento nell'ignota dimensione in cui Dante intravvede le anime avvolte dall'oscurità:

e l'occhio riposato intorno mossi,/ dritto levato, e fiso riguardai/per conoscer lo loco dov' io fossi./ Vero è che 'n su la proda mi trovai/ de la valle d'abisso dolorosa che 'ntrono accoglie d'infiniti guai./ Oscura e profonda era e nebulosa/ tanto che, per ficcar lo viso a fondo,/ io non vi discernea alcuna cosa". (If IV, vv.4-12).

Nel finale della scena delle selezioni infatti si legge: 
LA LINGUA PER NARRARE L'ASSURDO

Una guida alla lingua di Se questo è un uomo di Primo Levi

Anna Basevi

Scomparvero così, in un istante, a tradimento, le nostre donne, i nostrigenitori, i nostri figli.

Li vedemmo un po' di tempo come una massa oscura all'altra estremità della banchina, poi non vedemmopiù nulla". (p. 13).

I latrati, invece, vengono uditi anche in un altro incontro della Divina Commedia:

Cerbero, fiera crudele e diversa,/ contre gole caninamente latra/ sovra la gente che quivi è sommersa. (If VI, vv.13-15).

In Levi il suono del tedesco urlato dalle SS rende tale lingua diversa dal tedesco conosciuto, è la lingua di un altro universo, una dimensione ignota e inaspettata:

Tutto era incomprensibile e folle. (p. 14).

I quattro parlano una lingua che non sembra di questo mondo. ( $p$. 16).

Una lingua che addirittura ha l'effetto fisico di stravolgere l'espressione di chi la usa o la deve tradurre, come se le parole di cui è composta provocassero un gusto insopportabile in bocca:

Si apre la porta, entra um tedesco. È il maresciallo di prima;parla breve, l'interprete traduce (...).Si vedono le parole non sue, Le parole cattive, torcergli la boccauscendo, come se sputasse un boccone disgustoso. (p. 17).

La prima figura esplicitamente modellata su uno stereotipo dantesco che incontriamo è Caronte, al termine del primo capitolo, subito dopo la selezione, ed è rappresentata da un soldato tedesco che, durante il trasposto su un autocarro, chiede orologi e soldi ai prigionieri mentre vengono condotti in un regno di oscurità:

È un soldatotedesco, irto d'armi: non lo vediamo perché è buio fitto, ma ne sentiamo il contattoduro ogni volta che uno scossone del veicolo ci getta tutti in un mucchio a destra o asinistra. Accende una pila tascabile, e invece di gridare 'Guai a voi, anime prave' cidomanda cortesemente ad uno ad uno, in tedesco e in lingua franca, se abbiamo danaro od 
LA LINGUA PER NARRARE L'ASSURDO

Una guida alla lingua di Se questo è un nomo di Primo Levi

Anna Basevi

orologi da cedergli: tanto dopo non ci servono più. Non è un comando, non è regolamento questo: si vede bene che è una piccola iniziativa privata del nostro caronte. (p. 14).

Anche per Dante si tratta di uno dei primi incontri, situato nel terzo canto ma nel suo caso accompagnato da grida e minacce:

Ed ecco verso noi venir per nave/ un vecchio, bianco per antico pelo,/ gridando: 'Guai a voi, anime prave!/ Non isperate mai veder lo cielo:/ i' vegno per menarvi a l'altra riva/ ne le tenebre etterne, in caldo e 'n gelo'. (If III, vv.84-87).

Caron dimonio, con occhi di bragia/loro accennando, tutte le raccoglie;/batte col remo qualunque s'adagia". (If III, vv. 109-111).

Dopo le selezioni e il trasporto con il Caronte teutonico, i deportati arrivano alla porta della città infernale:

Il viaggio non durò che una ventina di minuti. Poi l'autocarro si è

fermato, e si è vista una grande porta, e sopra una scritta vivamente illuminata (ilsuo ricordo ancora mi percuote nei sogni): ARBEIT MACHT FREI, il lavoro rendeliberi. (p.15).

Le parole tedesche, se pur grottesche perche paradossali,suonano come un inquietante ammonimento. L'accostamento con i versi di Dante si impone con tale forza da indurci a leggere la scritta "Arbeit macht frei" quasi come la traduzione di "Lasciate ogni speranza voi che entrate":

Per me si va ne la città dolente,/per me si va ne l'etterno dolore,/ per me si va tra la perduta gente. [...]. (If III, vv.1-3).

Dinanzi a me non fuor cose create/se non eterne, e io etterno duro/ Lasciate ogne speranza, voich'intrate'./Queste parole di colore oscuro/vid' io scritte al sommo d'unaporta". (IF III, vv.7-9).

Che siamo entrati nella citta dolente, tra la perduta gente si confermerà da subito. Al primo buio della prima giornata,i nuovi arrivati girano tra la folla, alla ricerca di un viso amico, di un orientamento. Il prigioniero Levi incontra un ragazzo polacco con cui imbastisce uno stentato e breve dialogo col poco 
LA LINGUA PER NARRARE L'ASSURDO

Una guida alla lingua di Se questo è un nomo di Primo Levi

Anna Basevi

tedesco a sua disposizione. Lo ricorda come un momento luminoso di contatto umano:

Non ho più rivisto Schlome, ma non ho dimenticato il suo volto grave e mite di fanciullo che mi ha accolto sulla soglia della casa dei morti. (p. 23).

Per ciò che concerne questo brano, è necessario sottolineare la corrispondenza intertestuale con Dostoievski, messa in luce ed analizzata da Alberto Cavaglion, di cui si segnala, oltre alle note in SQU, il saggio Il senso dell'arca, Ebrei senza saperlo: nuove riflessioni. ${ }^{31}$

c) Prestiti danteschi

Tra i prestiti danteschi ci si imbatte nei concetti e nelle immagini di generale conoscenza e che assumono funzioni metaforiche, quali il limbo:

La vita del $\mathrm{Ka}-\mathrm{Be}^{32}$ è vita di limbo. I disagi materiali sono relativamente pochi, a parte la fame e le sofferenze inerenti alle malattie. Non fa freddo e non si lavora,e, a meno di commettere qualche grave mancanza, non si viene percossi. (p. 40).

il contrappasso:

Il traffico coi civili è (...) d'altronde un reato(...); viene perciò punito con particolare severità. (...)lo stesso lavoratore civile (...) può venire denunziato (...) e condannato a trascorrere (...) nelle stesse nostre condizioni un periodo variabile (...) dai quindici giorni agli otto mesi. Gli operai a cui viene applicato questo genere di contrappasso, vengono come noi spogliati all'ingresso. (p. 70).

la bolgia:

la bolgia buia e urlante del Block. (p. 102).

\footnotetext{
${ }^{31}$ CAVAGLION, Alberto. Il senso dell'arca, Ebrei senza saperlo: nuove riflessioni. Napoli: L'ancora, 2006. Si veda specialmente il capitolo "Sulla soglia della casa dei morti", p. 75-82

${ }^{32} \mathrm{Ka}-\mathrm{Be}$ era l' infermeria del Lager dove i prigionieri erano momentaneamente esenti dalle fatiche del lavoro forzato, e quindi anche dalle percosse e dal freddo.
} 
LA LINGUA PER NARRARE L'ASSURDO

Una guida alla lingua di Se questo è un nomo di Primo Levi

Anna Basevi

Alex vola gli scalini: ha le scarpe di cuoio perché non è ebreo, è leggero sui piedi

come i diavoli di Malebolge.(p. 93).

la schiera:

Regolarmente, a partire dalle tredici in punto, il cantiere si svuota e la schiera grigia interminabilesfila per due ore davanti alle due stazioni di controllo, dove come ogni giorno veniamo contati e ricontati.(...) Siamo noi, grigie identici, piccoli come formiche e grandi fino alle stelle. (p. 110).

Alberto ed io camminiamo spalla contro spalla nella lunga schiera grigia, curvi in avanti per resistere meglio al vento. (p. 126).

Anche l'importante e centrale metafora dei sommersi $^{33}$ viene ripresa da Dante come si vede nel verso già citato: "Cerbero, fiera crudele e diversa,/ con tre gole caninamente latra/ sovra la gente che quivi è sommersa".

d) Il tempo dell' eterno dolore e il dolore del ricordare il tempo felice

Un' ultimo parallelismo che vorremmo sottolineare riguarda l' idea già approfondita del tempo eterno. Ricordiamo che la sensazione dei prigionieri del Lager era di un tempo bloccato, stagnante, di cui non si vedeva la fine, come un aspetto dell' essere espropriati della vita, un tempo che "passa goccia a goccia" ${ }^{34}$ e che non prevede nemmeno un futuro:

Sapete come si dice 'mai' nel gergo del campo? 'Morgen früh', domani mattina.(p. 116).

Per noi il Lager non è una punizione; per noi non è previsto untermine, e il Lager altro non è che il genere di esistenza a noi

\footnotetext{
${ }^{33}$ I sommersi e $i$ salvati era il titolo che Primo Levi voleva per la sua prima opera, intitolata invece SQU sotto suggerimento dell' editore. Tuttavia e' stato usato come titolo di uno dei capitoli di SQU e dell' ultimo libro, un saggio che ritorna al tema ddel Lager e chiude la produzione dello scrittore, morto l' anno successivo. Con il termine "sommersi" Levi ha voluto indicare tutti coloro che non sono tornati, in opposizione ai "salvati", cioe' i superstiti, sviluppando importanti analisi e riflessioni sui motivi delle sorti degli uni e degli altri.

${ }^{34}$ SQU, p. 15
} 
LA LINGUA PER NARRARE L'ASSURDO

Una guida alla lingua di Se questo è un uomo di Primo Levi

Anna Basevi

assegnato, senzalimiti di tempo, in seno all'organismo sociale germanico.(p. 71).

Come non ricordare l'eternità dannata del celebre inizio del terzo canto ("Per me si va ne l'etterno dolore,/ per me si va tra la perduta gente./ Dinanzi a me nonfuor cose create/se non eterne, e io etterno duro").

$\mathrm{Al}$ contempo, in quest' eternità di sofferenza nemmeno i ricordi giovano e ristorano, come spiega Francesca a Dante nei celebri versi:

E quella a me: 'Nessun maggior/ dolore che ricordarsi del tempo felice/ ne la miseria; e ciò sa il tuo dottore'. (If V vv.121-123).

Tramite una finzione, il poeta fiorentino aveva colto un sentimento molto verosimile, che si rinnova nel Lager, allorquando ricordare tempi felici duole ancor più che dimenticarli:

Avevamo deciso di trovarci, noi italiani, ogni domenicasera in un angolo del Lager; ma abbiamo subito smesso, perché era troppo tristecontarci, e trovarci ogni volta più pochi, e più deformi, e più squallidi. Ed era cosifaticoso fare quei pochi passi: e poi, a ritrovarsi, accadeva di ricordare e dipensare, ed era meglio non farlo(p. 28)

\section{e) Citazioni}

Nonostante tanta presenza dell'inferno dantesco, le citazioni dirette vere e proprie - se si esclude il capitolo "Il canto di Ulisse"- sono poche.Ne troviamo una in un brano in cui si descrive la fisionomia del prigioniero del campo.Un'immagine che fa degli Häftlinge ("prigionieri", in tedesco) i dannati dei tempi moderni in un mondo di morte e sofferenza di cui, come abbiamo visto, l'Inferno di Dante si impone come forte metafora:

Dopo quindici giorni dall’ingresso, già ho la fame regolamentare $[. .$.$] Già$ mi sono apparse, sul dorso dei piedi, le piaghe torpide che non guariranno. Spingo vagoni, lavoro di pala, mi fiacco alla pioggia, tremo al vento; già il mio stesso corpo non è più mio: ho il ventre gonfio e le membra stecchite, il viso tumido al mattino e incavato a sera; qualcuno 
LA LINGUA PER NARRARE L'ASSURDO

Una guida alla lingua di Se questo è un uomo di Primo Levi

Anna Basevi

fra noi ha la pelle gialla, qualche altro grigia: quando non ci vediamo per tre o quattro giorni, stentiamo a riconoscerci l'un l'altro. (p. 28)

La citazione appartiene al sesto canto dell'Inferno:

Voi cittadini mi chiamaste Ciacco:/per la dannosa colpa de la gola,/come tu

vedi, a la pioggia mi fiacco. (If VI, vv.52-54).

Da notare che le due situazioni vengono unite sia dalla citazione sulla pioggia sia dal tema del mangiare : il dannato Ciacco si fiacca alla pioggia per scontare il peccato di gola, mentre Levi soffre di fame cronica.

A parte questi, tra $\mathrm{i}$ versi danteschi citati per esteso da Primo Levi troviamo solo:

Qui non ha luogo il Santo Volto/qui si nuota altrimenti che nel Serchio!(If XXI, vv.48-49 citati in SQU a p. 21).

\section{Lo maggior corno della fiamma antica}

Parleremo succintamente del capitolo "Il canto di Ulisse", molto studiato e citato ${ }^{35}$. In esso, dopo un antefatto che contestualizza l'episodio, vediamo il prigioniero Levi camminare verso le cucine con il compagno di prigionia Jean, un alsaziano soprannominato Pikolo $^{36}$, incaricato del trasporto del cibo e che quel giorno sceglie Primo come aiutante. Un'occasione unica di dialogo, giacché all'andata si va leggeri senza pesi. Conversando delle loro case e madri, l'amico francese cerca di allungare il percorso e esprime la voglia di

\footnotetext{
${ }^{35}$ Per altre analisi critiche nostre e di altri sul capitolo "Il canto di Ulisse" e ulteriore bibliografia cfr. Dissertação de Mestrado di BASEVI, Anna. A lingua que salva. Babel e Literatura em Primo Levi.(UFRJ, 2012)

${ }^{36}$ Jean Samuel, sopravvissuto, e` rimasto poi in contatto con Primo Levi ed e` autore del libro autobiografico: SAMUEL, Jean.Mi chiamava Pikolo. Milano: Frassinelli, 2008
} 
imparare l'italiano. Levi propone di cogliere l'attimo. "Non possiamo farlo? Possiamo. Anche subito (...) l'importante è non sprecare quest'ora". ${ }^{37}$

Ed ecco che sorge dalla memoria il canto di Ulisse, "chissà come e perché", ma "non abbiamo tempo di scegliere". "Jean è attentissimo, ed io comincio, lento ed accurato: Lo maggior corno della fiamma antica".

Si impone, quindi, una lingua infuocata che vuol parlare di sé e per di più - è interessante sottolinearlo - nella sua lingua madre allo stato, per così dire, più concentrato o originario, cioè attraverso l'italiano trecentesco. Assistiamo al doppio sforzo di ricordare i versi imparati a scuola e il tentativo di tradurre in francese la seconda parte del ventiseiesimo canto dell'Inferno dantesco (noto anche come "Canto di Ulisse", appunto) .

L'italiano Primo tenta inoltre di spiegarlo e commentarlo. Nel farlo inciampa in lacune, ma una gran fretta lo sospinge per poter arrivare al nucleo del canto:

\section{Considerate la vostra semenza}

Fatti non foste a viver come bruti,

Ma per seguir virtute e conoscenza. (If XXVI, vv. 118-120).

Ad Auschwitz Levi-Ulisse ${ }^{38}$ fa il suo compagno “arguto" e lo incita a meditare e nel farlo incontra egli stesso significati nascosti, risposte linguistiche illuminanti .

In questa frenetica e singhiozzante lezione che lo avvicina alla patria, alla lingua madre, alla relazione letteratura-vita, sarebbe disposto al massimo sacrificio per riuscire a ricostruire l'intera sequenza poetica; eppure la memoria vacilla e Primo invoca:"Darei la zuppa di oggi per saldare 'non ne avevo

\footnotetext{
${ }^{37}$ SQU, tutto il capitolo, p. 95-100

${ }^{38}$ Si veda per un'analisi della figura di Ulisse nella letteratura BOITANI, Piero. L'ombra di Ulisse.Bologna: Il Mulino, 1992, e sul Canto di Ulisse di Primo Levi le p. 183-188.
} 
alcuna' col finale".E a tratti, pur nello sforzo del ricordo e nella precarietà della traduzione che si rivela "un disastro", si illumina di fronte a significati svelati dalla realtà del campo:"qualcosa di gigantesco che io stesso ho visto ora soltanto".

Ma è tardi, stanno per arrivare, sono arrivati: l'esperienza si chiude come un brusco risveglio, allorquando risuonano le parole straniere e quotidiane "Kraut und Ruben" (Cavoli e rape). Allora la realtà del Lager li sommerge di nuovo e il capitolo si chiude con il verso finale del Canto XXVI con l'immagine potente del naufragio:

Infin che ' 1 mar fu sopra noi rinchiuso.

La scelta del canto appare quindi spontanea ma non casuale: l'Ulisse dantesco è una figura mitica particolare, diversa da quella omerica. Innanzitutto il terribile naufragio finale fa da eco alla prigionia come anticamera dello sterminio. Inoltre, lo spirito di Ulisse si impone prepotentemente per l'atteggiamento relativo alla comprensione, all' avventura e al superamento degli orizzonti del sapere umano. Nel lager, le colonne d'Ercole sono in forma di filo spinato e tale sete di "virtute e conoscenza" finisce per sprofondare l' eroe negli abissi; eppure, malgrado il finale tragico, Ulisse rimane l'eroe "umano" che affila le armi della conoscenza e della comprensione sia per tirarsi fuori dalle difficoltà, sia per nutrire la curiosità delle "cose umane" esattamente come Levi.

E come Ulisse, lo scrittore affina gli strumentidella parola, della comunicazione, del capire per resistere all' esproprio della propria identità, attingendo alla cultura come patrimonio vivo e nutrimento, come legame con le proprie radici.

"Il canto di Ulisse" costituisce proprio il momento in cui si addensa il rapporto con il multilinguismo e la traduzione,da un lato, (come mezzo di 
LA LINGUA PER NARRARE L'ASSURDO

Una guida alla lingua di Se questo è un nomo di Primo Levi

Anna Basevi

comunicazione e quindi di resistenza) e, dall'altro, con la propria lingua, quest'ultima come appiglio che guida verso una via di sopravvivenza.

Proprio in riferimento al tentativo di ricordare i versi di Dante nel Lager, lo scrittore affermò in seguito:"A me la cultura è stata utile; non sempre, a volte forse per vie sotterranee ed impreviste, ma mi ha servito e forse salvato.(...)Avrei dato veramente pane e zuppa, cioè sangue, per salvare dal nulla quei ricordi. ${ }^{39} \mathrm{E}$ inoltre:

Prima e dopo "Ulisse", ricordo di aver ossessionato i miei compagni italiani perché mi aiutassero a recuperare questo o quel brandello del mio mondo di ieri (..) leggendo nei loro occhi fastidio e sospetto:che cosa va cercando questo qui, con Leopardi e il "Numero di Avogadro" Che la fame non lo stia facendo diventar matto?.(SES, p. 113).

Era invece "un modo di ritrovare me stesso", aggiunge Levi.

Come si è detto, Levi sa di rischiare per compiere un atto preciso di cultura e di comunicazione umana, proprio quella negata dai nazisti nei lager poiché il campo di concentramento è una negazione del dialogo dei detenuti con il mondo, con le loro radici, con i loro paesi di provenienza, con la vita stessa. Ma darebbe il pane (equivalente alla garanzia di vita di quel giorno) per riuscire a ricordare tutto il canto, legare un verso a un altro.

Inoltre si somma lo sforzo di tradurre e spiegare in francese. Anche tradurre rappresenta un allargamento dei confini culturali e del sapere, un atto di comunicazione estremo che getta un ponte tra lingue, tra culture, tra esseri umani. Così come ricordare chi si era o si è, pur sotto gli stracci rigati, nel Lager-Babele, costruito per rendere tutti dei sordomuti destinati a morire, ritornare alla sorgente della propria identità linguistica e culturale significa tenere saldo quel filo che potrà portare all'uscita dal labirinto-Auschwitz.Il

\footnotetext{
${ }^{39}$ LEVI, Primo. I sommersi e i salvati. Torino: Einaudi, 2007 (1 ed.1987), p. 112. Citeremo con SES il titolo del testo.
} 
capitolo dell'utopica esegesi del canto di Ulisse intrapresa in quel contesto di disumanizzazione e schiavitù danno all'opera una profondità tanto letteraria quanto umana e ne fanno uno dei capitoli più commentati e citati di Se questo è un nomo.

L'atto letterario, quindi,attraverso le scelte linguistiche e stilistiche che ne costituiscono ingredienti essenziali, permette, rinvigorisce e solidifica l'intento del testimone di rappresentare attraverso la narrazione un'esperienza che molti hanno taciuto per decenni o che la società, uscita dai disastri della guerra, ha tentato di non ascoltare per molto tempo. Ma il testo rivela soprattutto un grande scrittore, oggi consacrato nel panorama letterario italiano come uno dei classici del secondo Novecento più letti (anche nella scuola italiana media e superiore). Nonostante SQU sia stato tradotto in numerosissime lingue, l'uscita imminente della traduzione in lingua inglese delle opere complete presso l'editore statunitense Norton attirerà certamente nuovi interessi e studi su tutta la produzione letteraria e saggistica di Primo Levi.

\section{Riferimenti Bibliografici}

BELPOLITI, Marco. Primo Levi. Milano: Mondadori, 1998, p. 107

LEVI, Primo. Se questo è un nomo. Torino: Einaudi, 2012, edizione commentata a cura di Alberto Cavaglion.

MENGALDO, Pier Vincenzo. Lingua e scrittura in Levi. In: FERRERO, Ernesto (org.). Primo Levi: un'antologia della critica. Torino: Einaudi, 1997, p. 197. 